\title{
Introduction to Neuro-Fuzzy Systems
}

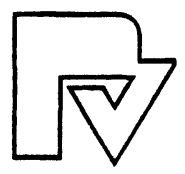




\section{Advances in Soft Computing}

\section{Editor-in-chief}

Prof. Janusz Kacprzyk

Systems Research Institute

Polish Academy of Sciences

ul. Newelska 6

01-447 Warsaw, Poland

E-mail: kacprzyk@ibspan.waw.pl

Esko Turunen

Mathematics Behind Fuzzy Logic

1999. ISBN 3-7908-1221-8 


\section{Robert Fullér}

\section{Introduction to}

Neuro-Fuzzy Systems

With 185 Figures

and 11 Tables

Springer-Verlag Berlin Heidelberg GmbH 
Prof. Robert Fullér

Department of Operations Research

Eötvös Lorànd University

Rakoczi ut 5

1088 Budapest

Hungary

Email: rfuller@cs.elte.hu

and

Institute of Advanced Management Systems Research

Åbo Akademi University

Lemminkäisenkatu 14B

2520 Turku

Finland

Email: rfuller@ra.abo.fi

ISBN 978-3-7908-1256-5

Cataloging-in-Publication Data applied for

Die Deutsche Bibliothek - CIP-Einheitsaufnahme

Fullér, Robert: Introduction to neuro-fuzzy systems / Robert Fullér. -

Springer-Verlag Berlin Heidelberg 2000

(Advances in soft computing)

$$
\begin{aligned}
& \text { ISBN 978-3-7908-1256-5 } \\
& \text { DOI 10.1007/978-3-7908-1852-9 }
\end{aligned}
$$

This work is subject to copyright. All rights are reserved, whether the whole or part of the material is concerned, specifically the rights of translation, reprinting, reuse of illustrations, recitation, broadcasting, reproduction on microfilm or in any other way, and storage in data banks. Duplication of this publication or parts thereof is permitted only under the provisions of the German Copyright Law of September 9, 1965, in its current version, and permissinon for use must always be obtained formSpringer-Verlag Berlin Heidelberg. Violations are liable for prosecution under the German Copyroght Law.

C Springer-Verlag Berlin Heidelberg 2000

Originally published by Physica-Verlag Heidelberg in 2000

The use of general descriptive names, registered names, trademarks, etc. in this publication does not imply, even in the absence of a specific statement, that such names are exempt from the relevant protective laws and regulations and therefore free for general use.

\section{Softcover Design: Erich Kirchner, Heidelberg}

SPIN 10747248

88/2202-5 43210 - Printed on acid-free paper 


\section{Preface}

Fuzzy sets were introduced by Zadeh (1965) as a means of representing and manipulating data that was not precise, but rather fuzzy. Fuzzy logic provides an inference morphology that enables approximate human reasoning capabilities to be applied to knowledge-based systems. The theory of fuzzy logic provides a mathematical strength to capture the uncertainties associated with human cognitive processes, such as thinking and reasoning. The conventional approaches to knowledge representation lack the means for representating the meaning of fuzzy concepts. As a consequence, the approaches based on first order logic and classical probablity theory do not provide an appropriate conceptual framework for dealing with the representation of commonsense knowledge, since such knowledge is by its nature both lexically imprecise and noncategorical.

The developement of fuzzy logic was motivated in large measure by the need for a conceptual framework which can address the issue of uncertainty and lexical imprecision.

Some of the essential characteristics of fuzzy logic relate to the following [242].

- In fuzzy logic, exact reasoning is viewed as a limiting case of approximate reasoning.

- In fuzzy logic, everything is a matter of degree.

- In fuzzy logic, knowledge is interpreted a collection of elastic or, equivalently, fuzzy constraint on a collection of variables.

- Inference is viewed as a process of propagation of elastic constraints.

- Any logical system can be fuzzified.

There are two main characteristics of fuzzy systems that give them better performance for specific applications.

- Fuzzy systems are suitable for uncertain or approximate reasoning, especially for the system with a mathematical model that is difficult to derive.

- Fuzzy logic allows decision making with estimated values under incomplete or uncertain information. 
Artificial neural systems can be considered as simplified mathematical models of brain-like systems and they function as parallel distributed computing networks. However, in contrast to conventional computers, which are programmed to perform specific task, most neural networks must be taught, or trained. They can learn new associations, new functional dependencies and new patterns.

The study of brain-style computation has its roots over 50 years ago in the work of McCulloch and Pitts (1943) and slightly later in Hebb's famous Organization of Behavior (1949). The early work in artificial intelligence was torn between those who believed that intelligent systems could best be built on computers modeled after brains, and those like Minsky and Papert who believed that intelligence was fundamentally symbol processing of the kind readily modeled on the von Neumann computer. For a variety of reasons, the symbol-processing approach became the dominant theme in artifcial intelligence. The 1980s showed a rebirth in interest in neural computing: Hopfield (1985) provided the mathematical foundation for understanding the dynamics of an important class of networks; Rumelhart and McClelland (1986) introduced the backpropagation learning algorithm for complex, multi-layer networks and thereby provided an answer to one of the most severe criticisms of the original perceptron work.

Perhaps the most important advantage of neural networks is their adaptivity. Neural networks can automatically adjust their weights to optimize their behavior as pattern recognizers, decision makers, system controllers, predictors, etc. Adaptivity allows the neural network to perform well even when the environment or the system being controlled varies over time. There are many control problems that can benefit from continual nonlinear modeling and adaptation.

While fuzzy logic performs an inference mechanism under cognitive uncertainty, computational neural networks offer exciting advantages, such as learning, adaptation, fault-tolerance, parallelism and generalization. A brief comparative study between fuzzy systems and neural networks in their operations in the context of knowledge acquisition, uncertainty, reasoning and adaptation is presented in the following table [93]:

To enable a system to deal with cognitive uncertainties in a manner more like humans, one may incorporate the concept of fuzzy logic into the neural networks. The resulting hybrid system is called fuzzy neural, neural fuzzy, neuro-fuzzy or fuzzy-neuro network.

Neural networks are used to tune membership functions of fuzzy systems that are employed as decision-making systems for controlling equipment. Although fuzzy logic can encode expert knowledge directly using rules with linguistic labels, it usually takes a lot of time to design and tune the membership functions which quantitatively define these linquistic labels. Neural network learning techniques can automate this process and substantially reduce development time and cost while improving performance. 


\begin{tabular}{|c|c|c|c|}
\hline Skills & & Fuzzy Systems & Neural Nets \\
\hline $\begin{array}{l}\text { Knowledge } \\
\text { acquisition }\end{array}$ & $\begin{array}{l}\text { Inputs } \\
\text { Tools }\end{array}$ & $\begin{array}{l}\text { Human experts } \\
\text { Interaction }\end{array}$ & $\begin{array}{l}\text { Sample sets } \\
\text { Algorithms }\end{array}$ \\
\hline Uncertainty & $\begin{array}{l}\text { Information } \\
\text { Cognition }\end{array}$ & $\begin{array}{l}\text { Quantitive and } \\
\text { Qualitive } \\
\text { Decision making }\end{array}$ & Quantitive \\
\hline Reasoning & $\begin{array}{l}\text { Mechanism } \\
\text { Speed }\end{array}$ & $\begin{array}{l}\text { Heuristic search } \\
\text { Low }\end{array}$ & $\begin{array}{l}\text { Parallel computat. } \\
\text { High }\end{array}$ \\
\hline Adaption & $\begin{array}{l}\text { Fault-tolerance } \\
\text { Learning }\end{array}$ & $\begin{array}{l}\text { Low } \\
\text { Induction }\end{array}$ & $\begin{array}{l}\text { Very high } \\
\text { Adjusting weights }\end{array}$ \\
\hline $\begin{array}{l}\text { Natural } \\
\text { language }\end{array}$ & $\begin{array}{l}\text { Implementation } \\
\text { Flexibility }\end{array}$ & $\begin{array}{l}\text { Explicit } \\
\text { High }\end{array}$ & $\begin{array}{l}\text { Implicit } \\
\text { Low }\end{array}$ \\
\hline
\end{tabular}

Table 0.1. Properties of fuzzy systems and neural networks.

In theory, neural networks, and fuzzy systems are equivalent in that they are convertible, yet in practice each has its own advantages and disadvantages. For neural networks, the knowledge is automatically acquired by the backpropagation algorithm, but the learning process is relatively slow and analysis of the trained network is difficult (black box). Neither is it possible to extract structural knowledge (rules) from the trained neural network, nor can we integrate special information about the problem into the neural network in order to simplify the learning procedure.

Fuzzy systems are more favorable in that their behavior can be explained based on fuzzy rules and thus their performance can be adjusted by tuning the rules. But since, in general, knowledge acquisition is difficult and also the universe of discourse of each input variable needs to be divided into several intervals, applications of fuzzy systems are restricted to the fields where expert knowledge is available and the number of input variables is small.

To overcome the problem of knowledge acquisition, neural networks are extended to automatically extract fuzzy rules from numerical data.

Cooperative approaches use neural networks to optimize certain parameters of an ordinary fuzzy system, or to preprocess data and extract fuzzy (control) rules from data.

The basic processing elements of neural networks are called artificial neurons, or simply neurons. The signal flow from of neuron inputs, $x_{j}$, is considered to be unidirectionalas indicated by arrows, as is a neuron's output signal flow. Consider a simple neural net in Figure 0.1. All signals and weights are real numbers. The input neurons do not change the input signals so their output is the same as their input. The signal $x_{i}$ interacts with the weight $w_{i}$ to produce the product $p_{i}=w_{i} x_{i}, i=1, \ldots, n$. The input information $p_{i}$ is 
aggregated, by addition, to produce the input

$$
\text { net }=p_{1}+\cdots+p_{n}=w_{1} x_{1}+\cdots+w_{n} x_{n}
$$

to the neuron. The neuron uses its transfer function $f$, which could be a sigmoidal function,

$$
f(t)=\frac{1}{1+e^{-t}}
$$

to compute the output

$$
y=f(\text { net })=f\left(w_{1} x_{1}+\cdots+w_{n} x_{n}\right) .
$$

This simple neural net, which employs multiplication, addition, and sigmoidal $f$, will be called as regular (or standard) neural net.

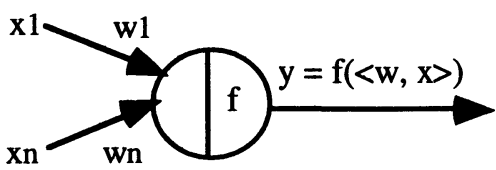

Fig. 0.1. A simple neural net.

If we employ other operations like a t-norm, or a t-conorm, to combine the incoming data to a neuron we obtain what we call a hybrid neural net. These modifications lead to a fuzzy neural architecture based on fuzzy arithmetic operations. A hybrid neural net may not use multiplication, addition, or a sigmoidal function (because the results of these operations are not necesserily are in the unit interval).

A hybrid neural net is a neural net with crisp signals and weights and crisp transfer function. However, (i) we can combine $x_{i}$ and $w_{i}$ using a tnorm, t-conorm, or some other continuous operation; (ii) we can aggregate the $p_{i}$ 's with a t-norm, t-conorm, or any other continuous function; (iii) $f$ can be any continuous function from input to output.

We emphasize here that all inputs, outputs and the weights of a hybrid neural net are real numbers taken from the unit interval $[0,1]$. A processing element of a hybrid neural net is called fuzzy neuron.

It is well-known that regular nets are universal approximators, i.e. they can approximate any continuous function on a compact set to arbitrary accuracy. In a discrete fuzzy expert system one inputs a discrete approximation to the fuzzy sets and obtains a discrete approximation to the output fuzzy set. Usually discrete fuzzy expert systems and fuzzy controllers are continuous mappings. Thus we can conclude that given a continuous fuzzy expert system, or continuous fuzzy controller, there is a regular net that can uniformly approximate it to any degree of accuracy on compact sets. The problem with 
this result that it is non-constructive and does not tell you how to build the net.

Hybrid neural nets can be used to implement fuzzy IF-THEN rules in a constructive way. Though hybrid neural nets can not use directly the standard error backpropagation algorithm for learning, they can be trained by steepest descent methods to learn the parameters of the membership functions representing the linguistic terms in the rules.

The direct fuzzification of conventional neural networks is to extend connection weigths and/or inputs and/or fuzzy desired outputs (or targets) to fuzzy numbers. This extension is summarized in the next table.

\begin{tabular}{clll}
\hline Fuzzy neural net & Weights & Inputs & Targets \\
\hline Type 1 & crisp & fuzzy & crisp \\
Type 2 & crisp & fuzzy & fuzzy \\
Type 3 & fuzzy & fuzzy & fuzzy \\
Type 4 & fuzzy & crisp & fuzzy \\
Type 5 & crisp & crisp & fuzzy \\
Type 6 & fuzzy & crisp & crisp \\
Type 7 & fuzzy & fuzzy & crisp \\
\hline
\end{tabular}

Table 0.2. Direct fuzzification of neural networks.

Fuzzy neural networks of Type 1 are used in classification problem of a fuzzy input vector to a crisp class. The networks of Type 2, 3 and 4 are used to implement fuzzy IF-THEN rules. However, the last three types in Table 0.2 are unrealistic.

- In Type 5, outputs are always real numbers because both inputs and weights are real numbers.

- In Type 6 and 7, the fuzzification of weights is not necessary because targets are real numbers.

$A$ regular fuzzy neural network is a neural network with fuzzy signals and/or fuzzy weights, sigmoidal transfer function and all the operations are defined by Zadeh's extension principle. Consider a simple regular fuzzy neural net in Figure 0.2 .

All signals and weights are fuzzy numbers. The input neurons do not change the input signals so their output is the same as their input. The signal $X_{i}$ interacts with the weight $W_{i}$ to produce the product $P_{i}=W_{i} X_{i}, i=$ $1, \ldots, n$, where we use the extension principle to compute $P_{i}$. The input information $P_{i}$ is aggregated, by standard extended addition, to produce the input 


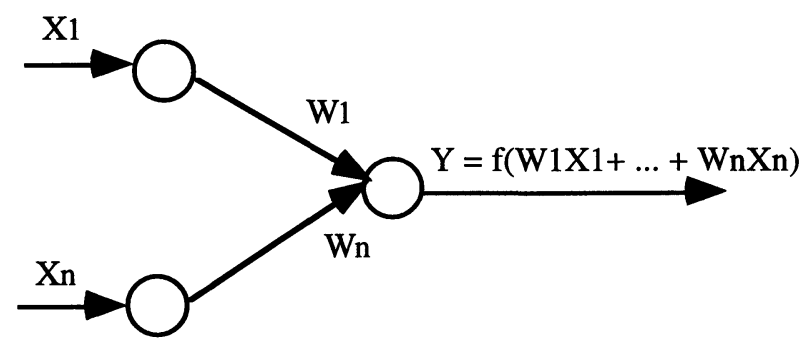

Fig. 0.2. Simple regular fuzzy neural net.

$$
\text { net }=P_{1}+\cdots+P_{n}=W_{1} X_{1}+\cdots+W_{n} X_{n}
$$

to the neuron. The neuron uses its transfer function $f$, which is a sigmoidal function, to compute the output

$$
Y=f(\text { net })=f\left(W_{1} X_{1}+\cdots+W_{n} X_{n}\right)
$$

where $f$ is a sigmoidal function and the membership function of the output fuzzy set $Y$ is computed by the extension principle.

The main disadvantage of regular fuzzy neural network that they are not universal approximators. Therefore we must abandon the extension principle if we are to obtain a universal approximator.

A hybrid fuzzy neural network is a neural network with fuzzy signals and/or fuzzy weights. However, (i) we can combine $X_{i}$ and $W_{i}$ using a tnorm, t-conorm, or some other continuous operation; (ii) we can aggregate the $P_{i}$ 's with a t-norm, t-conorm, or any other continuous function; (iii) $f$ can be any function from input to output.

Buckley and Hayashi [31] showed that hybrid fuzzy neural networks are universal approximators, i.e. they can approximate any continuous fuzzy functions on a compact domain.

This Lecture Notes is organized in four Chapters. The First Chapter is dealing with inference mechanisms in fuzzy expert systems. The Second Chapter provides a brief description of learning rules of feedforward multilayer supervised neural networks, and Kohonen's unsupervised learning algorithm for classification of input patterns. In the Third Chapter we explain the basic principles of fuzzy neural hybrid systems. In the Fourth Chapter we present a case study and some excercises for the Reader.

This book is a textbook on neuro-fuzzy systems used for a course taught by the author at $\AA$ bo Akademi University between 1995 and 1999. 


\section{Contents}

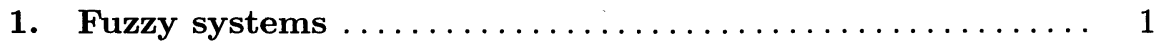

1.1 An introduction to fuzzy $\operatorname{logic} \ldots \ldots \ldots \ldots \ldots \ldots \ldots \ldots \ldots \ldots$

1.2 Operations on fuzzy sets $\ldots \ldots \ldots \ldots \ldots \ldots \ldots \ldots \ldots \ldots \ldots \ldots \ldots$

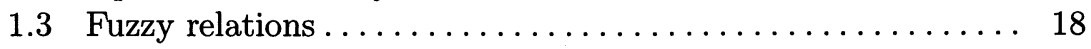

1.4 The extension principle $\ldots \ldots \ldots \ldots \ldots \ldots \ldots \ldots \ldots \ldots .26$

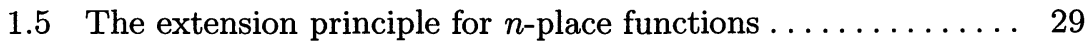

1.6 Metrics for fuzzy numbers $\ldots \ldots \ldots \ldots \ldots \ldots \ldots \ldots \ldots \ldots \ldots$

1.7 Measures of possibility and necessity .............. 41

1.8 Fuzzy implications . . . . . . . . . . . . . . . . . . . . 45

1.9 Linguistic variables $\ldots \ldots \ldots \ldots \ldots \ldots \ldots \ldots \ldots \ldots \ldots \ldots \ldots$

1.9.1 The linguistic variable Truth ............... 50

1.10 The theory of approximate reasoning............... 53

1.11 An introduction to fuzzy logic controllers ............. 71

1.12 Defuzzification methods . . . . . . . . . . . . . . . . . 78

1.13 Inference mechanisms . .................... 81

1.14 Construction of data base and rule base of FLC . . . . . . 86

1.15 The ball and beam problem ................... 91

1.16 Aggregation in fuzzy system modeling............... 95

1.17 Averaging operators $\ldots \ldots \ldots \ldots \ldots \ldots \ldots \ldots \ldots \ldots . \ldots 9$

1.18 Fuzzy screening systems . . . . . . . . . . . . . . . 109

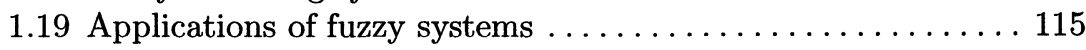

Bibliography ................................. 119

2. Artificial neural networks . ...................... 133

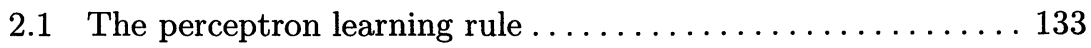

2.2 The delta learning rule $\ldots \ldots \ldots \ldots \ldots \ldots \ldots \ldots \ldots \ldots \ldots$

2.3 The delta learning rule with semilinear activation function ... 149

2.4 The generalized delta learning rule ................ 154

2.5 Effectivity of neural networks $\ldots \ldots \ldots \ldots \ldots \ldots \ldots \ldots \ldots \ldots \ldots$

2.6 Winner-take-all learning . ...................... 160

2.7 Applications of artificial neural networks . . . . . . . . . 164

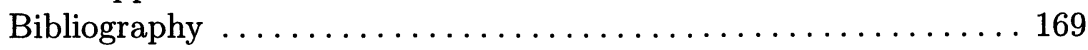


3. Fuzzy neural networks $\ldots \ldots \ldots \ldots \ldots \ldots \ldots \ldots \ldots \ldots \ldots \ldots \ldots \ldots \ldots$

3.1 Integration of fuzzy logic and neural networks . . . . . . . 171

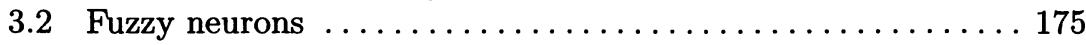

3.3 Hybrid neural nets. . . . . . . . . . . . . . . . . 184

3.4 Computation of fuzzy logic inferences by hybrid neural net . 195

3.5 Trainable neural nets for fuzzy IF-THEN rules . . . . . . . 201

3.6 Implementation of fuzzy rules by regular FNN of Type 2.... 208

3.7 Implementation of fuzzy rules by regular FNN of Type $3 \ldots 212$

3.8 Tuning fuzzy control parameters by neural nets $\ldots \ldots \ldots \ldots 216$

3.9 Fuzzy rule extraction from numerical data . . . . . . . . . 224

3.10 Neuro-fuzzy classifiers . . . . . . . . . . . . . . . . . . . 228

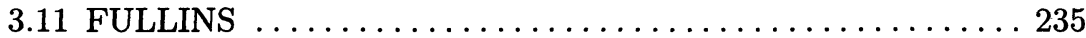

3.12 Applications of fuzzy neural systems $\ldots \ldots \ldots \ldots \ldots \ldots 240$

Bibliography ............................... 245

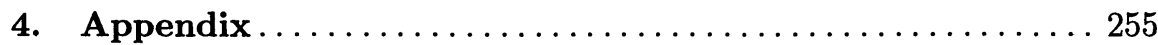

4.1 Case study: A portfolio problem ................. 255

4.1.1 Tuning the membership functions $\ldots \ldots \ldots \ldots \ldots \ldots 259$

4.2 Exercises ................................ 262

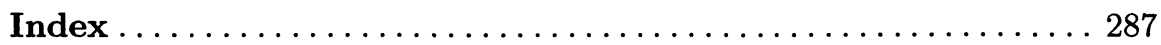

